\title{
Separation of isoclinics and isochromatics from photoelastic data with a regularized phase-tracking technique
}

\author{
J. A. Quiroga and A. González-Cano
}

\begin{abstract}
We present the application of a regularization algorithm to the processing of photoelastic fringe patterns. The method used is a modified regularized phase-tracking (RPT) algorithm applied to phase-shifted images. In particular, we present an algorithm for isoclinic-isochromatic separation that uses only five images. In the case of isoclinics the method can deal with problems associated with modulation of isochromatics and with isotropic points by means of a modified cost functional. With respect to the isochromatics the problems associated with regions of high fringe density are solved in a robust way by the unmodified RPT algorithm by use of the modulation information. The performance of the method is discussed, and experimental results are presented. (C) 2000 Optical Society of America
\end{abstract}

OCIS codes: $100.2650,120.5050,260.5430$.

\section{Introduction}

In recent years a big effort has been made to develop automatic methods for extracting information about photoelastic data. The main problem for all these techniques is the separation of the information associated with isoclinics and isochromatics. For that goal phase-measuring techniques have been applied. ${ }^{1-10}$ For a complete isoclinic-isochromatic phase recovery many images are required (three or four for isoclinics and six-eight for isochromatics) for performing the complete analysis and separating the fringe patterns. This is a drawback in some situations: For instance, in the method of threedimensional stress analysis of Plouzenec et al., 11 it is difficult to obtain the necessary number of images to apply phase-shifting techniques; however, it is well known that, in the phase-shifting technique, the fewer the images used, the more sensitive the corre-

J. A. Quiroga and A. González-Cano are with the Departamento de Óptica, Facultad de Ciencias Físicas, Universidad Complutense Cuidad Universitaria s/n, 28040 Madrid, Spain. When this research was performed, J. A. Quiroga (aq@fis.ucm.es) was with the Centro de Investigaciones en Optica, Loma del Bosque 115, Col. Lomas del Campestre, 37150 Leon, Guanajuato, Mexico. A. González-Cano's e-mail address is agus@fis.ucm.es.

Received 23 September 1999; revised manuscript received 3 February 2000.

0003-6935/00/172931-10\$15.00/0

(C) 2000 Optical Society of America sponding method is to errors such as nonsinusoidal patterns, nonlinearity in the detector, errors in the phase shifts, and background correction.

Also, there exist some specific problems associated with fringe patterns appearing in the photoelastic technique. One is that isoclinic and isochromatic fringe patterns are completely mixed. The modulation of the isoclinic phase map depends on the isochromatics and vice versa. Another problem is the isotropic points, which are points whose difference between principal stresses is zero. The isoclinic angle is not defined for these points, and the practical consequence of this is that at these points the isoclinic phase map presents low modulation, making further processing difficult. However, isochromatics usually present zones of high fringe density owing to high stress concentrations, for example, near the points where a force is applied. The consequence is that, because of sampling, aliasing or loss of modulation appear. For these reasons the unwrapping of the corresponding phase maps is difficult, owing to the appearance of logical inconsistencies associated with those low-modulation areas.

There exist in the literature several phasesampling-based strategies for isoclinic computation. They are used with the so-called linear or circular configurations of the polariscope. By an appropriate selection of the orientations of the elements of the polariscope several images are acquired (say, $I_{i}$ and 
$I_{j}$ ), the sine and the cosine of the isoclinic angle are computed as

$$
\begin{aligned}
& \sin (K \alpha)=\sum I_{i}, \\
& \cos (K \alpha)=\sum I_{j},
\end{aligned}
$$

where $K$ is a constant that depends on the polariscope configuration used. $K$ can be either 2 or 4 . From the sine and the cosine terms the isoclinic phase map is computed as

$$
W(K \alpha)=\arctan \left(\sum I_{i} / \sum I_{j}\right),
$$

where $W($ ) is the so-called wrapping operator indicating that the phase map computed by Eq. (3) is defined only in the range $[-\pi, \pi]$.

As we see below, the problem of Eq. (3) is that the terms in $\sin (K \alpha)$ and $\cos (K \alpha)$ are modulated by the isochromatic fringe pattern. For the linear configuration of the polariscope this modulation is proportional to $\sin ^{2}(\delta / 2)$, where $\delta$ is the isochromatic parameter, and, for the circular configuration of the polariscope, the modulation is proportional to $\sin (\delta)$. This means that the phase map computed by Eq. (3) is affected by low-modulation areas that produce logical inconsistencies and/or sudden sign jumps that complicate the subsequent processing of the isoclinic phase map, especially the phase unwrapping.

There exist several procedures to correct this problem. The first group can be classified as interpolation procedures (see, for example, Ref. 9). In this approach low-modulation areas are detected, and the corresponding values for the isoclinic phase map are interpolated from the high-modulated neighborhood. This idea presents some practical drawbacks. First, this process requires that the region to be processed should be as small as possible. The image to be interpolated is a phase map, which is discontinuous by definition, so interpolation is not easy when the region to be processed contains a phase jump. This can be the case if a low-modulation area is coincident with an isoclinic fringe. This fact can be alleviated if instead of interpolating the phase map directly we interpolate the sine and the cosine terms given by Eqs. (1) and (2), but even in this case, if the region to be interpolated contains a phase jump, a considerable distortion appears. Also, if the area to be interpolated lies on the border of the image, the interpolation process becomes extrapolation. And, finally, the thresholding of the modulation to detect the lowquality areas represents a compromise: A high value for the threshold is a secure procedure to select the valid points, but it can reject too many points; in contrast, a low value for the threshold assumes that many points are valid, so the interpolation is easy; but some of the points can still be affected by the low modulation present in their surroundings.

A second approximation uses the spectral depen- dence of the isochromatic parameter, which under elastic approximation is given by

$$
\delta=(2 \pi / \lambda) C\left(\sigma_{1}-\sigma_{2}\right),
$$

where $\lambda$ is the wavelength used, $C$ is the so-called photoelastic constant of the material, and $\sigma_{1}$ and $\sigma_{2}$ are the two principal stresses. As we stated above, the modulation is directly dependent on the isochromatic parameter, so if we are able to change the wavelength of the monochromatic light used, we will find that low-modulation areas move with the wavelength. Then, if we work with two or three wavelengths, we will obtain two or three isoclinic phase maps, with different regions of low quality and, by combining them, will be able to obtain an isoclinic phase map that is almost free of the defects produced by the isochromatics. ${ }^{5}$ One problem with this procedure is the increasing number of images used, three or four by each wavelength. This problem can be overcome if, instead of working with several wavelengths, we use a polychromatic light source. ${ }^{7}$ The continuous spectral contents of the polychromatic source will produce an integrating effect over the isochromatics, thus increasing the overall modulation. With this technique an additional white-light source is needed, and success depends on the spectral transmittance of the sample. Additionally, these spectral techniques cannot determine the isoclinic parameter at points placed on the zero-order isochromatic fringe, that is, points that are almost free of stress or isotropic points. So a small amount of extra interpolation can be necessary.

A related approach is so-called load stepping, ${ }^{10}$ in which, instead of using different wavelengths, we analyze the sample by using different loads, in this way making the regions of low-modulation move to select the isoclinic parameter in a manner similar to that of the three-wavelength method. Again, many images are needed (nine images in Ref. 10), and frozenstressed samples cannot be analyzed with load stepping. However, we can apply the mathematical scheme used for load stepping in Ref. 10 equivalently to a three-wavelength approach simply by changing the role of the loads and the wavelengths, thus enabling analysis of a frozen-stressed sample.

Once the isoclinic parameter is computed, there exist several good algorithms to calculate the isochromatic phase map. This is because, outside the high-density regions where aliasing and/or lack of modulation appear, the fringe pattern associated with isochromatics does not present difficulties as do the ones associated with the isotropic points in the case of isoclinics. Also, if the isoclinic parameter is known, it is possible to determine the two quadrature terms in sine and cosine of the isochromatic phase without any modulation, owing to the isoclinics, ${ }^{8}$ and then, by a common arctangent computation, determine the corresponding phase map. However, there remains the above-depicted problem of the high-stress regions, where the high density of fringes may produce aliasing or lack of 
modulation, which can produce phase maps that are difficult to demodulate.

To overcome these problems, in this study we propose application of a regularization $\operatorname{method}^{12}$ to the analysis of photoelastic fringe patterns. Regularization methods are based on the minimization of a cost functional where we take into account both the fidelity of the estimation to the experimental data (the fringe pattern, in our case) and a so-called regularization term that introduces some $a$ priori knowledge of the function to be recovered. These regularization schemes have been applied to direct phase extraction from single interferograms, ${ }^{13}$ phase unwrapping, ${ }^{14}$ and phase recovery from phase-shifted images. ${ }^{15}$ In particular we used a modified regularized phasetracking (RPT) algorithm. ${ }^{16}$ Especially in the case of phase-shifted images, the regularization methods have proved to be robust in both phase recovery and demodulation. In this way, applying the phasetracking method to the analysis of photoelastic fringe patterns, we will be able to recover with high quality the demodulated phase distributions associated with isoclinics and isochromatics from a reduced number of fringe patterns. The method can be applied to frozen-stressed samples.

This paper is organized as follows. In Section 2 the RPT algorithm is briefly outlined. In Section 3 the algorithm for the recovery of the isoclinic phase is presented. The algorithm for isochromatic computation is depicted in Section 4 . In Section 5 experimental results are presented; performance of the algorithm and its future improvements are discussed. Finally, in Section 6 conclusions are given.

\section{Regularized Phase-Tracking Algorithm}

The phase estimation of a fringe pattern may be formulated as an inverse problem that must be solved by an algorithm that incorporates some $a$ priori smoothness constraints about the phase being detected. To solve this problem, we may regularize it by proposing a suitable cost functional with two terms corresponding to, first, the fidelity between the estimated function and the observations, and second, the smoothness of the phase being detected. It is assumed that the estimated phase function is the minimizer of the proposed cost functional.

In the RPT technique developed by Servin et al. ${ }^{16}$ the fringe pattern is considered to be locally monochromatic; that is, the local irradiance is modeled as a cosinusoidal function phase modulated by a plane. The modeled sinusoidal function must be close to the irradiance of the pattern that corresponds to the fidelity term. In this way the phase must be adapted to every region in the pattern. Smoothness and continuity of the estimated phase are enforced by the regularization term. The cost functional proposed by Servin et al. to demodulate a pair of images in quadrature with no carrier is expressed as

$$
U_{T}=\sum_{x, y \in L} U_{x, y}\left(\phi, \omega_{x}, \omega_{y}\right)
$$

where

$$
\begin{aligned}
U_{x y}\left(\phi, \omega_{x}, \omega_{y}\right)= & \sum_{\xi \eta \in N \cap L}\left\{\left|f_{C}(\xi, \eta)-\cos \left[\phi_{e}(x, y, \xi, \eta)\right]\right|^{2}\right. \\
& +\left|f_{S}(\xi, \eta)-\sin \left[\phi_{e}(x, y, \xi, \eta)\right]\right|^{2} \\
& \left.+\lambda\left|\phi(\xi, \eta)-\phi_{e}(x, y, \xi, \eta)\right|^{2} m(\xi, \eta)\right\},
\end{aligned}
$$

$$
\begin{aligned}
\phi_{e}(x, y, \xi, \eta)= & \phi(x, y)+\omega_{x}(x, y)(x-\xi) \\
& +\omega_{y}(x, y)(y-\eta),
\end{aligned}
$$

where $U_{x, y}$ is the energy of the system at a site $(x, y)$ in the image, $L$ is a two-dimensional lattice that has valid data, and $N$ is a neighborhood region around the coordinate $(x, y)$ in the image where the phase is being determined. The field $m(x, y)$ is an indicator that equals 1 if the site has already been estimated and 0 otherwise. The fields $\omega_{x}$ and $\omega_{y}$ are the estimated local frequencies in the $x$ and the $y$ directions, respectively, which can be interpreted as the first derivatives of the phase. The so-called regularizing parameter $\lambda$ controls the smoothness of the detected phase, and $f_{C}(x, y)$ and $f_{S}(x, y)$ are the estimations of the cosine and the sine fringe patterns, respectively, associated with the phase to be computed, $\phi(x, y)$.

As Servin et al. mention, functional (5) is multimodal, so it may have several minima, which makes the minimization of this functional a difficult task. Following Servin et al., we compute a first estimation of the phase by minimizing the local cost functional (6) with the so-called crystal-growing algorithm, and, afterward, we improve the solution by applying the iterated conditional mode (ICM) algorithm depicted in Ref. 16. This algorithm is based on Bayesian estimation theory and is used to find local minima of complex energy functions. In our case we used the cost functional proposed by Servin et al. ${ }^{16}$ In that study the functional is chosen such that its minimization is equivalent to the application of the RPT when all the points have been estimated.

The key point of our algorithm is the use of a quality map that indicates whether a point is reliable. This quality map is obtained directly from the modulation of the fringe patterns as we show below. The crystal-growing process, by which every position $(x$, $y$ ) is visited, is controlled by this quality map so that the points with the highest quality are locally processed first, minimizing error propagation and extending the good results obtained in the high-quality regions.

With this purpose we used the quality-map-based algorithm described by Ströbel. ${ }^{17}$ Basically this algorithm sets up the quality map histogram and allocates memory for queue-type registers with every occurring quality value; thus the quality map must be quantized to a given number of levels. In our case the quality map is given in a natural way by the modulation of the fringe pattern being processed, as we see below. After the queue-type registers are filled with pixels according to their quality value, the processing starts with the pixel that has the highest- 
quality information; after this all adjacent (four connected) pixels of the start pixel are processed and entered into the registers according to their quality value. The pixel first entered from the highest nonempty register is taken, and its adjacent pixels are processed. This process is repeated until all the pixels have been processed (details can be found in Ref. 17).

\section{Isoclinic Computation}

The basic experimental setup for the photoelastic stress analysis is the so-called polariscope. The polariscope is used mainly in two different configurations, plane and circular. ${ }^{18}$ We used a circular polariscope consisting of a pair of linear polarizers, with two quarter-wave plates between them. The final polarizer is usually called the analyzer. The quarter-wave plates are placed after the polarizer and before the analyzer. The sample is placed between the quarter-wave plates. For isoclinic calculation the circular polariscope is used in a specific configuration, for which the incident light on the sample is linearly polarized. To achieve this, the fast axes of the quarter-wave plates must be parallel to the axes of the polarizers. If the polarizer and the analyzer are parallel, this is called a linear-brightfield configuration. We can obtain the intensity output for this configuration by applying Jones calculus, taking the sample as a linear retarder of retardation angle $\delta$, whose fast axis forms an angle $\alpha$ with the $x$ axis ( $\delta$ and $\alpha$ have a different value for each point of the sample). This output can be written as

$$
I_{\alpha}=I_{B}+I_{0} m_{\alpha} \cos [4(\alpha-\beta)]
$$

where

$$
\begin{aligned}
I_{B} & =I_{0}\left[1-\frac{1}{2} \sin ^{2}\left(\frac{\delta}{2}\right)\right], \\
m_{\alpha} & =\frac{1}{2} \sin ^{2}\left(\frac{\delta}{2}\right),
\end{aligned}
$$

are the background and the modulation, respectively, and $I_{0}$ is the input intensity. The term $\beta$ in Eq. (8) denotes an arbitrary phase shift that can be introduced by a rotation of the whole polariscope. Now we can take a set of three stepped images, $I_{\alpha 1}, I_{\alpha 2}$, and $I_{\alpha 3}$, by rotating the whole polariscope by a series of three angles, $\beta_{1}=0, \beta_{2}=2 \pi / 8$, and $\beta_{3}=3 \pi / 8$. From them we compute the background modulation and the input intensity as

$$
\begin{aligned}
I_{B} & =1 / 2\left(I_{\alpha 1}+I_{\alpha 2}\right), \\
m_{I} & =\left\{\left(I_{\alpha 1}-I_{\alpha 2}\right)^{2}+\left[2\left(I_{\alpha 3}-I_{B}\right)\right]^{2}\right\}^{1 / 2}, \\
I_{0} & =I_{B}+1 / 2 m_{I}, \\
m_{\alpha} & =1 / 2 \frac{m_{I}}{I_{0}}
\end{aligned}
$$

From Eqs. (8) and (14) we can compute the sine and the cosine terms to be demodulated as

$$
\begin{aligned}
& f_{\alpha C}=2 m_{\alpha} \cos (4 \alpha)=\frac{I_{\alpha 1}-I_{\alpha 2}}{I_{0}}, \\
& f_{\alpha S}=2 m_{\alpha} \sin (4 \alpha)=\frac{2\left(I_{\alpha 3}-I_{0}\right)}{I_{0}} .
\end{aligned}
$$

These two terms are introduced in Eq. (6), and the cost functional (5) is minimized in the function of $\phi$, $\omega_{x}$, and $\omega_{y}$, which in the case of isoclinic computation with the quadrature terms (15) and (16) correspond to $4 \alpha, 4 \partial \alpha / \partial x$, and $4 \partial \alpha / \partial y$, respectively.

As mentioned above, the key to successful demodulation of Eqs. (15) and (16) by the RPT algorithm is to visit the points in the lattice in the right way. In our case this process is controlled by a quality map that corresponds to the modulation term defined in Eq. (10) and computed by Eq. (14). In this way highmodulation areas are visited first, where the isoclinic parameter and its derivatives are computed in a safe way, and low-modulation areas are visited last, so the good values of their surroundings and the regularization term of the local cost functional (5) provide a regularized result over these areas. In this sense the RPT algorithm performs some kind of interpolation but with all the available information inside the area of interest weighted by the corresponding modulation.

This procedure is right if no isotropic points exist within the area of interest. As stated above, an isotropic point is a place where the difference between principal stresses is zero, which means that at that location the material behaves as isotropic, thus producing a singularity in the phase map associated with $4 \alpha$. For a first-order isotropic point this results in two close residues with the same sign; thus the local phase changes by $\pm 4 \pi$ rad as the point is encircled. The physical meaning of this is that as one encircles the first-order isotropic point the isoclinic angle $\alpha$ changes from 0 to $\pi$; as we are computing $4 \alpha$, we observe a total change of $4 \pi$. If the isotropic point is of the second order, the result is four close residues of the same sign; thus the local phase changes by $\pm 8 \pi \mathrm{rad}$ as the point is encircled, and so on. This means that, if an isotropic point is present, the $4 \alpha$ map is not continuous but presents a dislocation of $\pm 4 k \pi$, starting at the isotropic points.

This is an important problem for the algorithm depicted above for isoclinic computation: We know that the phase we wish to recover can be discontinuous (if an isotropic point is present); however, the regularization term of the local cost functional (6) imposes continuity. This is solved by introduction of a modification in the local cost functional (6). We know that, if there are isotropic points, the phase is going to have $\pm 4 k \pi$ jumps, so, if we calculate the differences appearing in the regularization term of (6) modulo $4 \pi$, we are going to recover the continuous-phase modulo $4 \pi$ associated with $4 \alpha$. Then, for our modified functional $m 4 \pi$, an $n 4 \pi$ rep- 
resents the same value for all $n, m$, being $n, m$ integers. The basic assumption of this modification is that no noise is present with an amplitude of $4 \pi \mathrm{rad}$ or greater. This is feasible in the case of photoelastic fringe patterns.

Finally, the modified local cost functional (6) becomes

$$
\begin{aligned}
U_{x y}\left(\phi, \omega_{x}, \omega_{y}\right)= & \sum_{\xi \eta \in N \cap L}\left\{\left|f_{\alpha C}(\xi, \eta)-\cos \left[\phi_{e}(x, y, \xi, \eta)\right]\right|^{2}\right. \\
& +\left|f_{\alpha S}(\xi, \eta)-\sin \left[\phi_{e}(x, y, \xi, \eta)\right]\right|^{2} \\
& +\lambda \mid W_{4 \pi}[\phi(\xi, \eta) \\
& \left.\left.-\phi_{e}(x, y, \xi, \eta)\right]\left.\right|^{2} m(\xi, \eta)\right\}
\end{aligned}
$$

where $W_{4 \pi}$ denotes the modulo- $4 \pi$ operation.

This procedure permits an automatic demodulation of the isoclinics with only three phase-stepped images in a robust way. Once the area of interest is defined, no extra masking is necessary, because the quality map provides all the information concerning the position of problematic points.

\section{Isochromatic Computation}

As we have said, there exist several good algorithms for computation of the phase map associated with isochromatics. The main problem is that highstress regions are not uncommon, which complicates further demodulation. This point is where the robust behavior of the RPT algorithm can help in this demodulation. In this case no modification of the local cost functional (6) is needed, so the standard formulation for demodulation with the RPT is used.

To compute isochromatics, monochromatic light illumination (a sodium spectral lamp) is used. Unlike isoclinics, isochromatics depends on the wavelength of the light source, the photoelastic constant, and the thickness of the sample. In this way isochromatics do not change with a rotation of the whole polariscope.

For simplicity we establish the following notation. We denote by $P, Q$, and $A$ the polarizer, the quarterwave plate, and the analyzer, respectively, and we specify the orientation of the elements by writing as a subscript the angle with respect to the $x$ axis formed by the transmission axis of the polarizers or the fast axis of the retardation plates. For instance, by $P_{90}$ we indicate a polarizer whose transmission axis is perpendicular to the chosen $x$ axis. We express the elements in the order in which the light finds them. The sample will be taken, as stated above, as a retardation plate of retardation $\delta$ whose fast axis forms an angle of $\alpha$ with the $x$ axis. For brevity the sample will not be included in the expression of the polariscope configurations.

Then, for instance, by $P_{90} Q_{45} Q_{(-45)} A_{0}$ we mean (i) a polarizer at $90^{\circ}$, (ii) a quarter-wave plate with fast axis at $45^{\circ}$, (iii) the sample (not explicitly specified), (iv) a quarter-wave plate with fast axis at $-45^{\circ}$, and (v) an analyzer at $0^{\circ}$.

For isoclinic computation two configurations of the

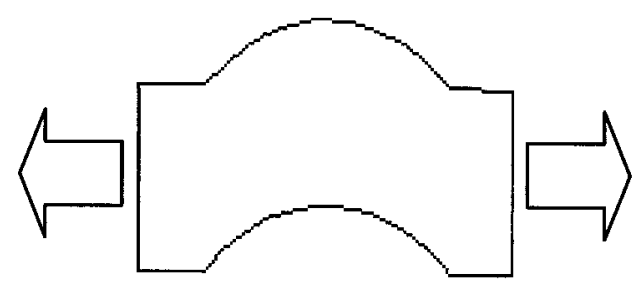

Fig. 1. Scheme of the first sample. Arrows indicate the sense and the direction of the applied force.

circular polariscope are used; they and their associated output intensities are given by

$$
\begin{gathered}
P_{90} Q_{45} Q_{45} A_{(-45)}: \quad I_{\delta 1}=\frac{I_{0}}{2}(1+\cos 2 \alpha \sin \delta), \\
P_{(-45)} Q_{90} Q_{90} A_{0}: \quad I_{\delta 2}=\frac{I_{0}}{2}(1+\sin 2 \alpha \sin \delta) .
\end{gathered}
$$

From the linear bright field configuration used to compute the isoclinics we can obtain $I_{0}$ by Eq. (13) and the term in $\sin ^{2}(\delta / 2)$ by Eq. (14). Then, applying Eqs. (13), (14), (19), and (20), we can compute the sine and the cosine terms to be demodulated as

$$
\begin{aligned}
& f_{\delta C}=\cos \delta=1-4 m_{\alpha}, \\
& f_{\delta S}=\sin \delta=\frac{\left[\left(2 I_{\delta 1}-I_{0}\right) \cos 2 \alpha+\left(2 I_{\delta 2}-I_{0}\right) \sin 2 \alpha\right]}{I_{0}} .
\end{aligned}
$$

In this case the quality map used to guide the demodulation of Eqs. (20) and (21) is calculated as

$$
m_{\delta}=\left(f_{\delta S}{ }^{2}+f_{\delta C}^{2}\right)^{1 / 2} \text {. }
$$

Again, these two terms are introduced in Eq. (6), and the cost functional (5) is minimized in the function of $\phi$, $\omega_{x}$, and $\omega_{y}$, which in the case of isochromatic computation with the quadrature terms (20) and (21), correspond to $\delta, \partial \delta / \partial x$, and $\partial \delta / \partial y$, respectively. The phase $\delta$ is recovered without any sign ambiguity out of an additive constant (piston term) that must be included if no isotropic points are present in the field of view. If an isotropic point is present, it is easy to locate it from the isoclinic map where it appears as a discontinuity. Isotropic points are always placed in the zero-order isochromatic fringe, so they provide a reference to determine the piston term. Thus, if an isotropic point is present in the field of view, the algorithm can perform an automatic complete retrieval of the isochromatic phase, once the processing mask is supplied.

\section{Experimental Results}

The first sample is a bar with an arc under axial traction, as shown schematically in Fig. 1, where the arrows indicate the sense and the direction of the applied force. The images corresponding to $f_{\alpha C}$ and $f_{\alpha S}$ [Eqs. (15) and (16)] are shown in Figs. 2(a) and 2(b), respectively; the modulation due to isochromatics can be clearly observed in the lower part of the arc. In the upper part of the arc it is possible to observe an iso- 


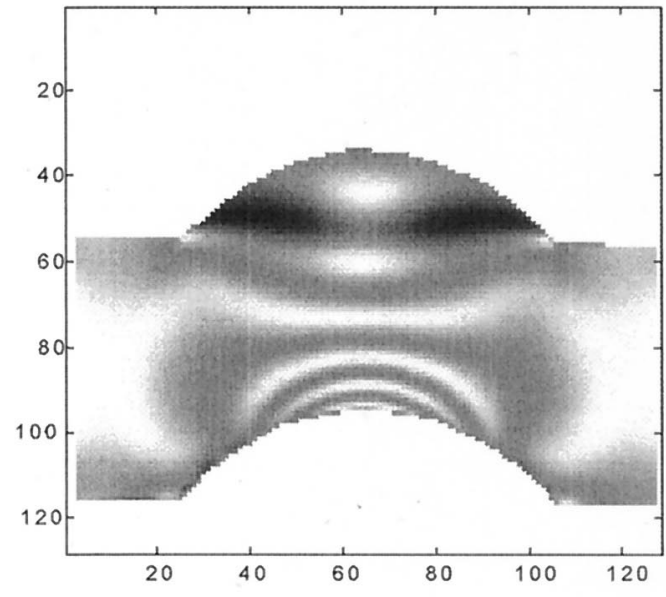

(a)

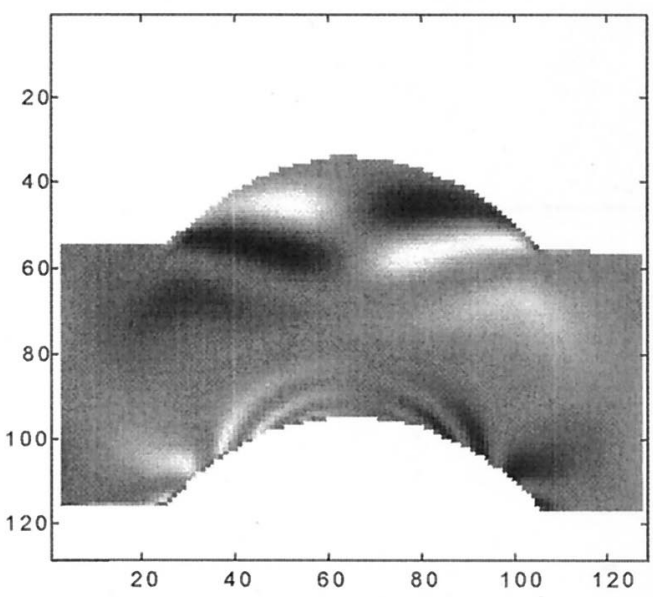

(b)

Fig. 2. Images corresponding to the cosine and the sine terms of the isoclinics, Eqs. (15) and (16).

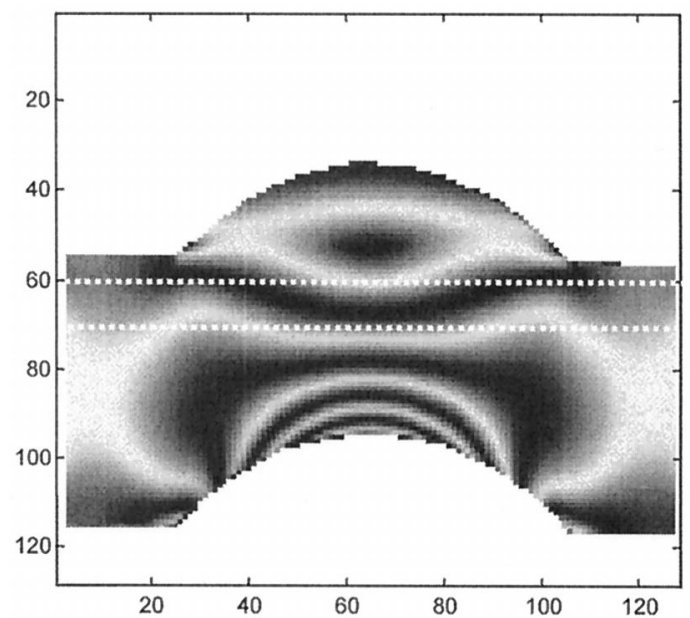

Fig. 3. Modulation map, $m_{\alpha}$, associated with Figs. 2(a) and 2(b).

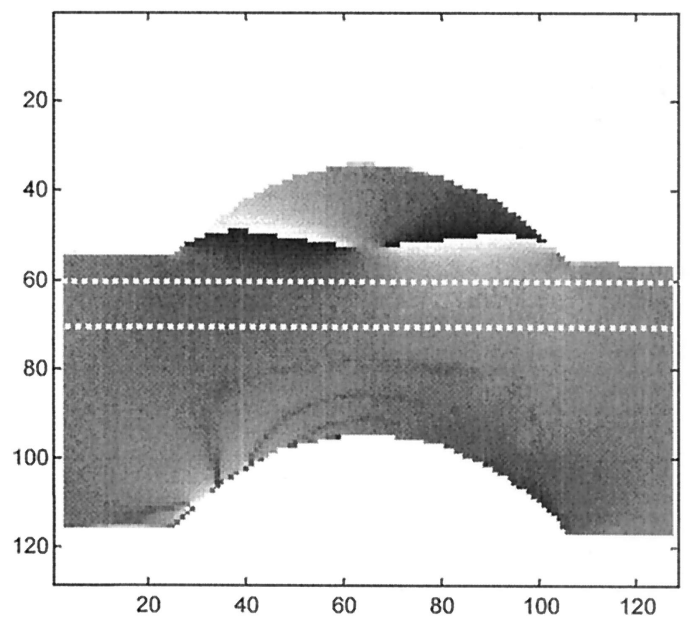

(a)

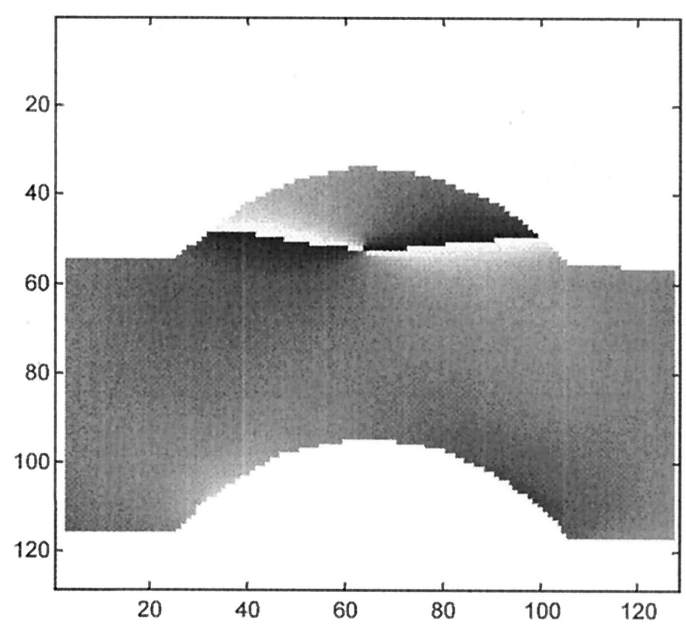

(b)

Fig. 4. (a) Isoclinic phase map obtained by the PS algorithm (see text), (b) isoclinic map obtained by the RPT algorithm. In these images black corresponds to $-\pi \mathrm{rad}$ and white to $\pi \mathrm{rad}$.

tropic point that is located at the zero-order isochromatic fringe. Figure 3 shows the quality map $m_{\alpha}$ given by Eq. (10) [that is, closely related to the term $f_{\delta C}$ of Eq. (20)]. In this figure black fringes represent areas of low modulation. Figure 4(a) shows the isoclinic phase $4 \alpha$ computed directly by the phasesampling (PS) formula as $W(4 \alpha)=\arctan \left(f_{\alpha S} / f_{\alpha C}\right)$; the areas of bad quality can be clearly observed in correspondence with the black fringes of Fig. 4. Figure 4(b) shows the continuous isoclinic phase calculated by the RPT technique. Here the image is shown wrapped between $[-\pi, \pi]$ for the sake of clarity, and in these figures black represents $-\pi \mathrm{rad}$ and white $\pi \mathrm{rad}$. Figures 5(a) and 5(b) show the profiles for both the RPT and the PS methods of the isoclinic angle along rows 60 and 70, respectively. These rows are sig- 


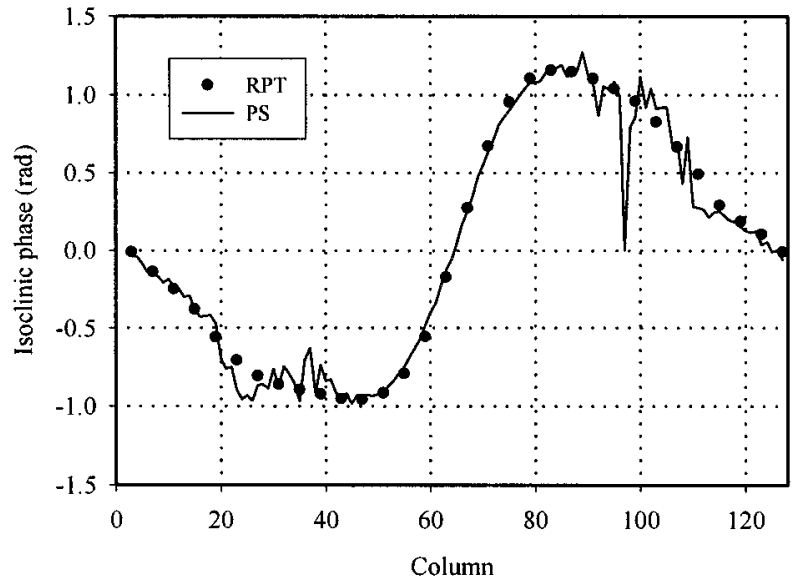

(a)

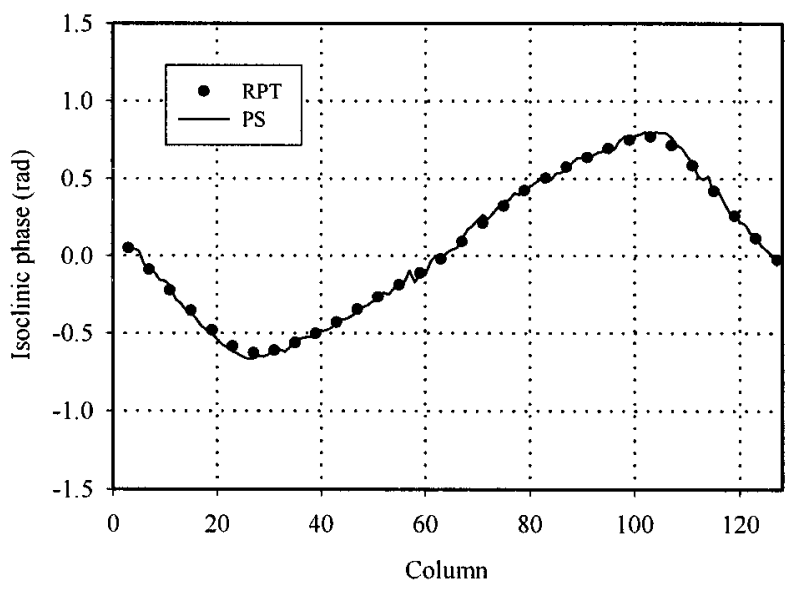

(b)

Fig. 5. Profiles of the isoclinic angle obtained by the PS and the RPT algorithms. The profiles have been taken along the dashed lines of Figs. 3 and 4(a). (a) Profile along line 60; the areas of low modulation are visible as fluctuation in the profile of the PS isoclinic angle. (b) Profile along line 70. Because the profile does not cross any low-modulation area, the results of the PS and the RPT algorithms almost coincide.

naled by two dashed lines in Figs. 3 and 4(a). These profiles show graphically the behavior of the RPT algorithm. Figure 3 shows that row 60 crosses a lowmodulation area two times; thus the PS procedure produces errors that can be observed in Fig. 4(a). The RPT technique reproduces the PS results in the areas of high modulation while filtering the results of the PS technique in the areas of low modulation. Figure 5(b) shows the profiles corresponding to row 70. As can be seen in Fig. 3, this row does not cross any lowmodulation area; thus the results of the PS and the

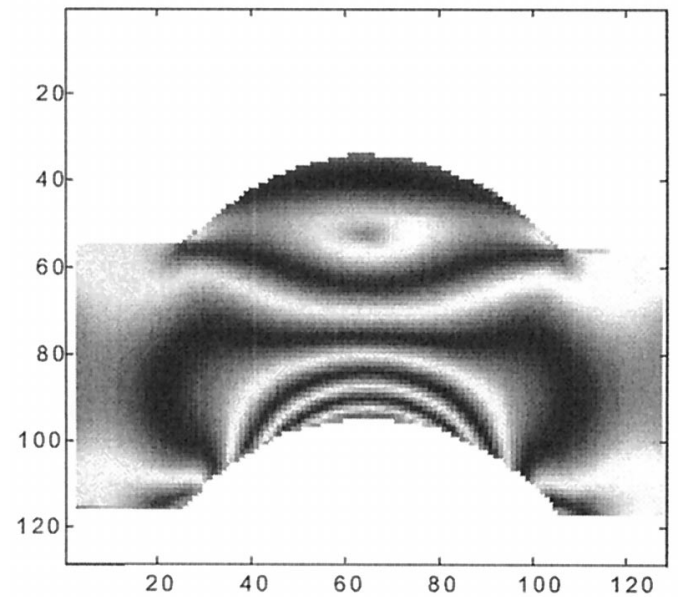

Fig. 6. Sine term of the isochromatics, Eq. (21), of the sample of Fig. 1.

RPT techniques almost coincide. In the isoclinic phase of Fig. 4(b) the location of the isotropic point is clear, which appears as a pair of discontinuities in the upper part of the arc. For the case of isoclinics this is a good example of how the algorithm can solve the problem of isotropic points.

Figure 6 shows the image corresponding to the $f_{\delta S}$ term [Eq. (21)], and in Fig. 7 the isochromatic phase computed by the RPT is shown wrapped modulo $2 \pi$ for the sake of clarity. In this figure black represents $-\pi$ rad and white $\pi$ rad. In this particular case it seems that the RPT algorithm offers no advantages over a conventional PS algorithm and a further demodulation, but we must remember that the RPT does not estimate only the phase but its gradients, too, and that they are estimated in a robust way. This fact is important if we wish to perform a further stress separation by integrating the equilibrium equations. ${ }^{19}$ The size of the images was $128 \times 128$. In the case of the isoclinics the value for the parameter $\lambda$ and the size of the neighborhood $N$, respectively, were 10 and $7 \times 7$ for the RPT iteration and 10 and $9 \times 9$ for the ICM

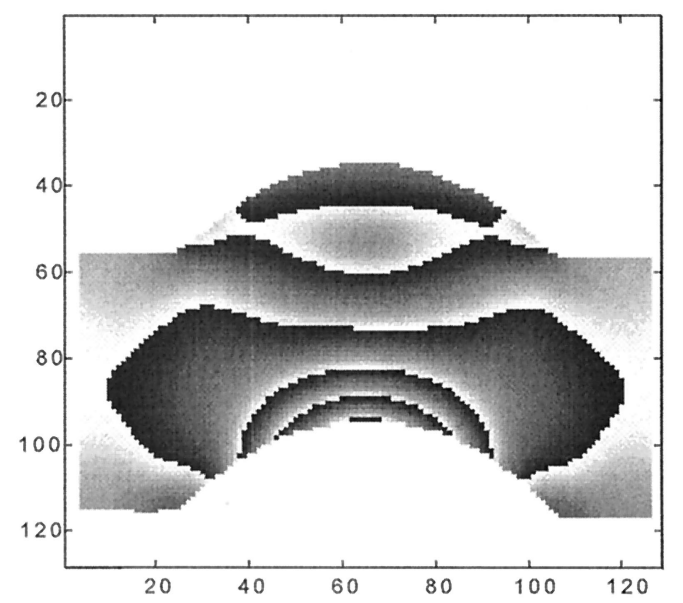

Fig. 7. Isochromatic phase map computed by the RPT technique. In this image black corresponds to $-\pi$ rad and white to $\pi$ rad. 


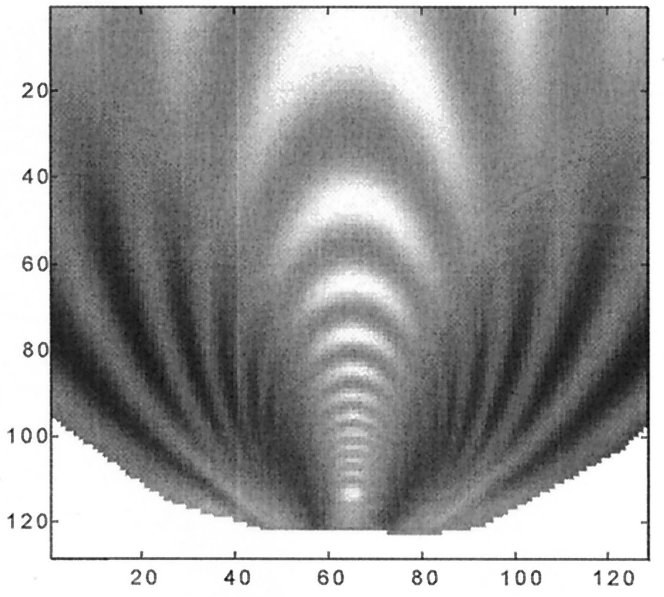

(a)

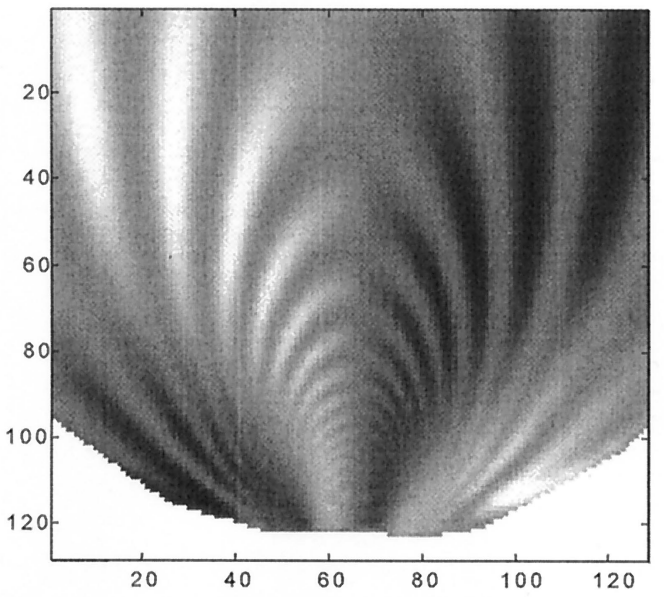

(b)

Fig. 8. (a) and (b) Images corresponding to the cosine and sine terms of the isoclinics, Eqs. (15) and (16), respectively, of a disk under diametral compression. In these images we observe the detail of the bottom part of the disk.

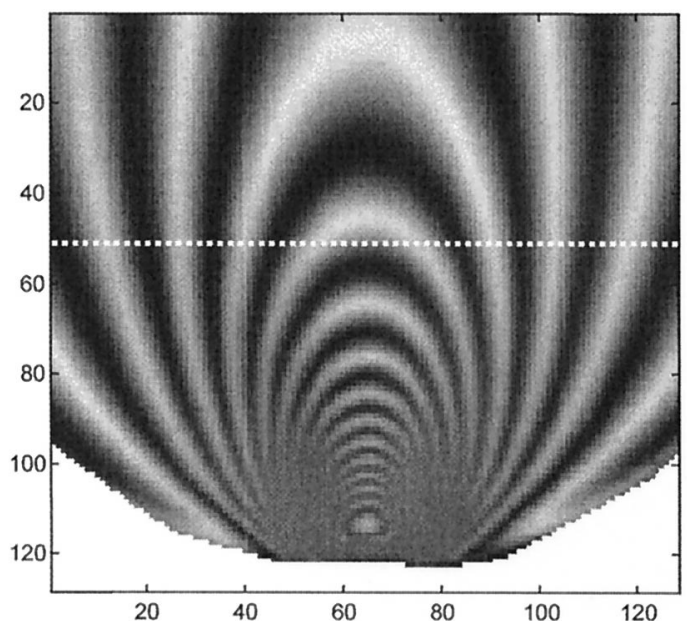

Fig. 9. Modulation map, $m_{\alpha}$, associated with images $8(a)$ and $8(b)$.

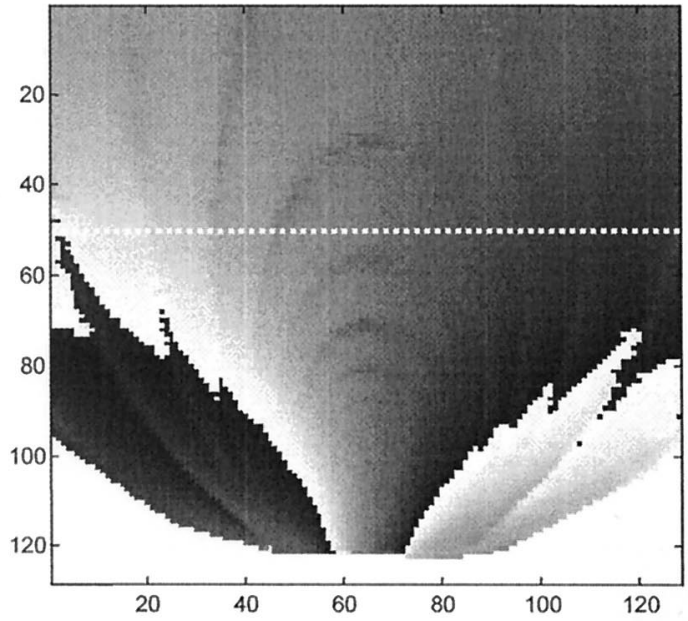

(a)

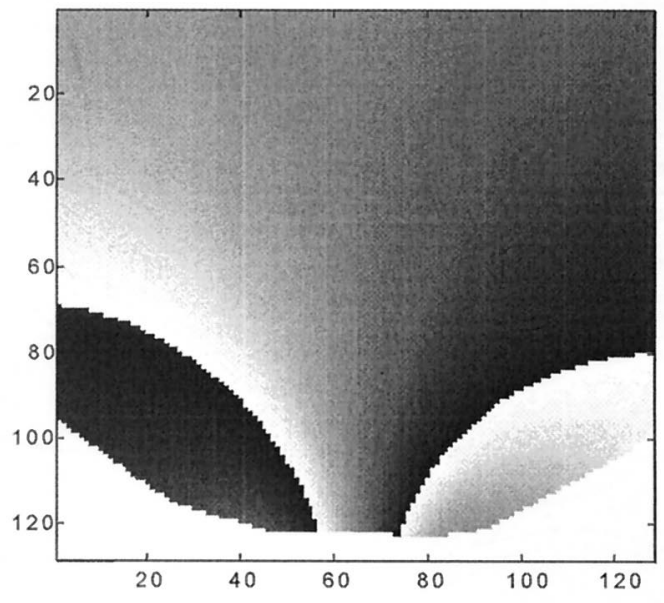

(b)

Fig. 10. (a) Isoclinic phase map obtained by the PS algorithm (see text), (b) isoclinic map obtained by the RPT algorithm. In these images black corresponds to $-\pi \mathrm{rad}$ and white to $\pi \mathrm{rad}$.

iteration. In the case of isochromatics the values were 1 and $3 \times 3$ for the RPT iteration and 5 and $5 \times$ 5 for the ICM iteration.

The second sample is a magnified part of a disk under diametrical compression. No difficulties associated with isotropic points appear in this case, but isochromatics have a high fringe density in the surroundings of the loading point, thus producing problems in calculation of the isoclinics and in the demodulation of isochromatics. The images corresponding to $f_{\alpha C}$ and $f_{\alpha S}$ [Eqs. (14) and (15)] are shown in Figs. 8(a) and 8(b), respectively. Figure 9 shows the quality map $m_{\alpha}$ given by Eq. (10). As above, black fringes represent areas of low modulation. Figure 10 (a) shows the isoclinic phase $4 \alpha$ computed directly by the PS method; the areas of bad quality can be clearly 


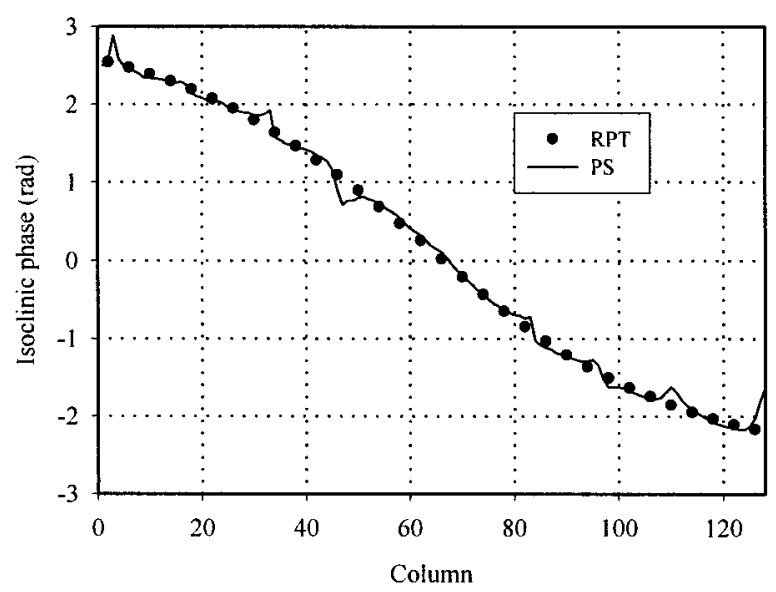

Fig. 11. Profile of the isoclinic angle obtained by the PS and the RPT algorithms. The profiles have been taken along the dashed lines of Figs. 9 and 10(a), which corresponds to line 50.

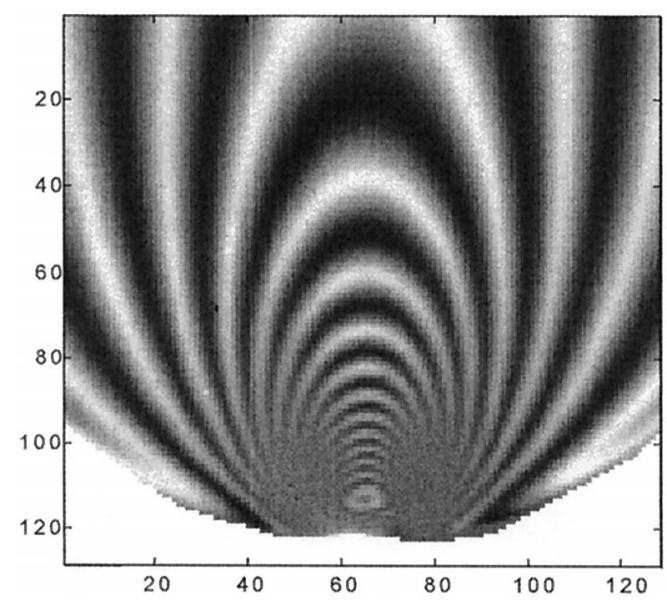

Fig. 12. Sine term of the isochromatics, Eq. (21), of the disk under diametral compression.

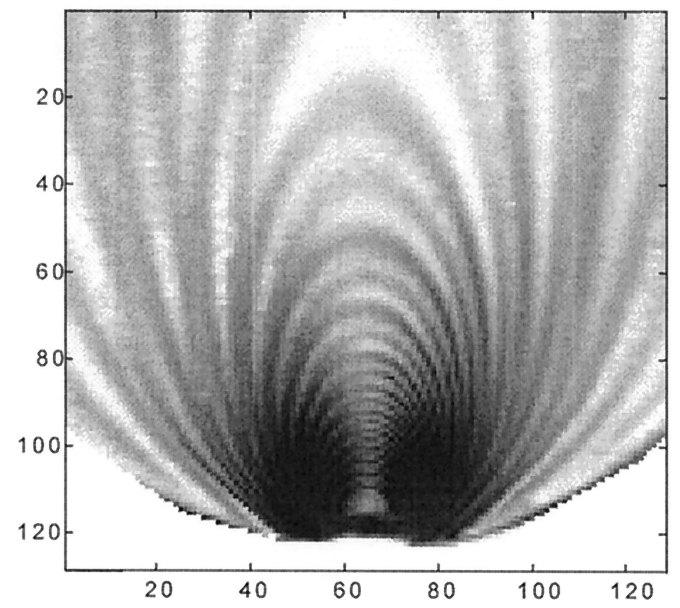

Fig. 13. Modulation map, $m_{\delta}$ Eq. (22), corresponding to the cosine and the sine terms shown in Figs. 9 and 12. observed in correspondence with the black fringes of Fig. 9. Figure 10(b) shows the continuous isoclinic phase calculated by the RPT technique; the image is shown wrapped between $[-\pi, \pi]$ for the sake of clarity. As above, in these figures black represents $-\pi$ rad and white $\pi \mathrm{rad}$. Figure 11 shows the profile for both the RPT and the PS methods of the isoclinic angle along row 50. This row is marked by a dashed line in Figs. 9 and 10(a). Again, as can be seen in Fig. 11, the RPT reproduces the results of the PS technique in the highmodulation areas while smoothing out the error produced by the low-modulation ones. Figure 12 shows the image corresponding to the $f_{\delta S}$ term [Eq. (21)], and the modulation map $m_{\delta}$ [Eq. (22)] is shown in Fig. 13. As can be seen, the areas of high fringe density in the

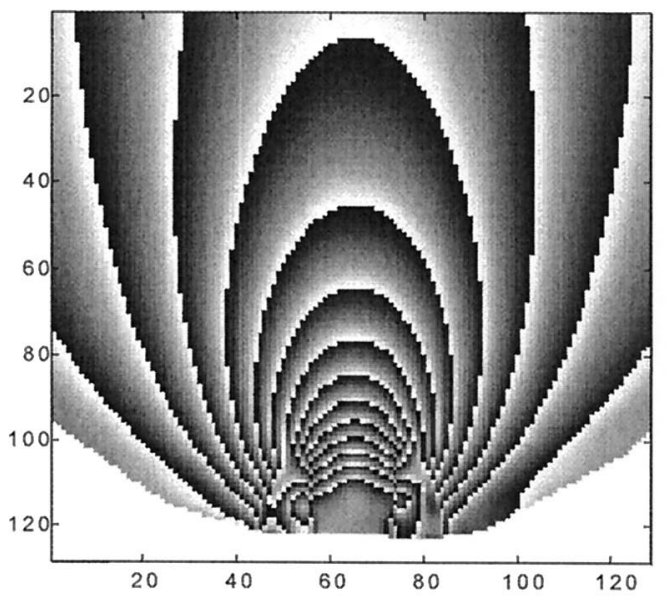

(a)

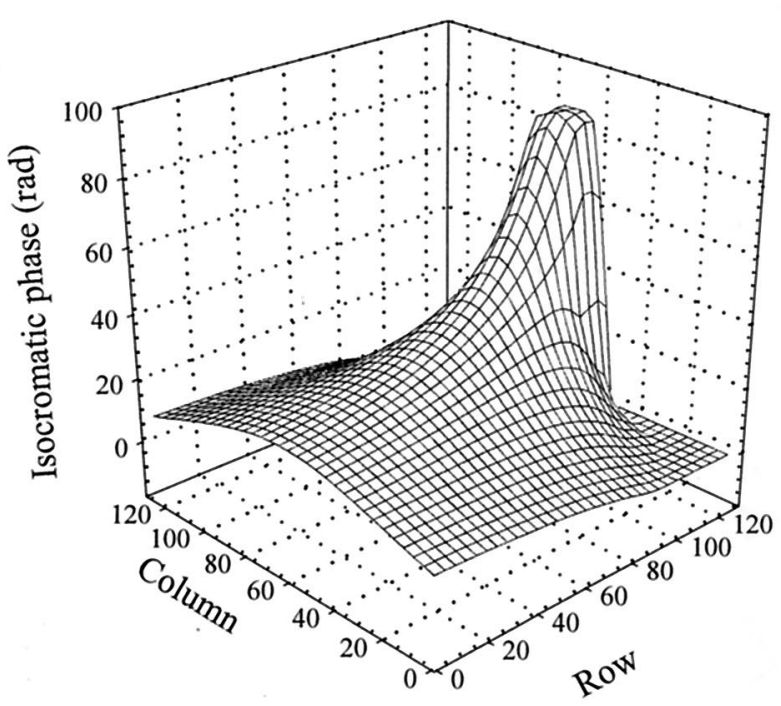

(b)

Fig. 14. (a) Isochromatic phase map computed by the RPT technique. In this image black corresponds to $-\pi \mathrm{rad}$ and white to $\pi$ rad. (b) Continuous isochromatic map computed by the RPT technique. 
bottom part of the disk produce a low-modulation area near the contact point. Figure 14(a) shows the continuous isochromatic phase computed by the RPT technique and wrapped between $[-\pi, \pi]$; the interpolation over the low-modulation areas is clear while no propagation of errors out of these areas appears. Finally a plot of the continuous isochromatic phase is shown in Figure 14(b). The size of the images is $128 \times 128$. In the case of the isoclinics the value for the parameter $\lambda$ and the size of the neighborhood $N$, respectively, were 10 and $17 \times 17$ for the RPT iteration and 10 and $19 \times$ 19 for the ICM iteration. In the case of isochromatics the values were 1 and $5 \times 5$ for the RPT iteration and 5 and $9 \times 9$ for the ICM iteration.

When the RPT technique is used, the processing time depends strongly on the minimization algorithm used. The gradient descent proposed by Servin et $a l .{ }^{16}$ can be successfully applied to recover the corresponding phase in simple cases, but in the case of photoelastic fringe patterns the poor convergence properties of the algorithm produce a bad phase recovery and noisy gradient fields. Taking this into account, we decided to use a more efficient minimization algorithm, that of Broyden-Fletcher-GoldfarbShanno. As we have shown, the performance of this algorithm with real images is good. However, the cost of the optimization algorithm robustness is a larger processing time. The implementation of this minimization algorithm is easy, because it is already included as a part of the optimization toolbox of the Matlab environment. ${ }^{20}$ In our case the processing time was $20 \mathrm{~min}$ for each iteration; then to compute one phase and two frequency fields, we need $\sim 40 \mathrm{~min}$ in a AMD K6-2 330-MHz computer equipped with 128 Mbyte of RAM. This processing time is greater by a factor of 10 than the conventional demodulation algorithms, but the robustness and the ability to process the complicated photoelastic fringe patterns makes RPT a good value. We are currently working on optimization of the processing time.

\section{Conclusions}

We have presented a regularization algorithm for processing photoelastic fringe patterns. The algorithm is a RPT with a modified local cost functional for isoclinics and with a path-following strategy based on the modulation. The algorithm uses five images for a complete demodulation of isoclinics and isochromatics, without sign ambiguity. Also, once the lattice of valid point masks is determined, the processing of the isoclinics and the isochromatics is done in an automatic way by means of the modulation information. We have successfully tested the algorithms with real images, proving that the performance of the algorithm is good for both isoclinics and isochromatics. The main drawback is the large processing time necessary to make a good estimation of the phase and its gradients.

During realization of this study J. A. Quiroga was supported in part by a post-doctoral grant from the
Universidad Complutense de Madrid and by the Becas Internacionales Flores-Valles program, Spain. We acknowledge the support of the Centro de Investigaciones en Óptica (CIO), León, Mexico.

\section{References}

1. E. A. Patterson and Z. F. Wang, "Towards full field automated photoelastic analysis of complex components," Strain 27, 49-53 (1991).

2. A. Asundi, "Phase shifting in photoelasticity," Exp. Tech. 17, 19-23 (1993).

3. J. Carazo-Alvarez, S. J. Haake, and E. A. Patterson, "Completely automated photoelastic fringe analysis," Opt. Lasers Eng. 21, 133-149 (1994).

4. Y. Morimoto, Y. Morimoto, and T. Hayashi, "Separation of isochromatics and isoclinics using Fourier transform," Exp. Tech. 13-17 (Sept./Oct. 1994).

5. C. Buckberry and D. Towers, "Automatic analysis of isochromatic and isoclinic fringes in photoelasticity using phasemeasuring techniques," Meas. Sci. Technol. 6, 1227-1235 (1995).

6. A. D. Nurse, "Full-field automated photoelasticity by use of a three-wavelength approach to phase stepping," Appl. Opt. 36, 5781-5786 (1997).

7. G. Petrucci, "Full-field automatic evaluation of an isoclinic parameter in white light," Exp. Mech. 37, 420-426 (1997).

8. J. A. Quiroga and A. González-Cano, "Phase measuring algorithm for extraction of isochromatics of photoelastic fringe patterns," Appl. Opt. 36, 8397-8402 (1997).

9. N. Plouzenec, J. C. Dupré, and A. Lagarde, "Whole field determination of isoclinic and isochromatic parameters," Exp. Tech. 30-33 (Jan./Feb. 1999)

10. M. J. Ekman and A. D. Nurse, "Completely automated determination of two-dimensional photoelastic parameters using load stepping," Opt. Eng. 37, 1845-1851 (1998).

11. N. Plouzenec, J. C. Dupré, and A. Lagarde, "Visualisation of photoelastic fringes within three dimensional specimens using an optical slicing method," in Proceedings of the IUTAM Symposium on Advanced Optical Methods and Applications in Solids Mechanics, A. Lagarde, ed. (Université de Poitiers, Poitiers, France, 1998), pp. P1-P8.

12. J. L. Marroquin, M. Rivera, S. Botello, R. Rodriguez-Vera, and M. Servin, "Regularization methods for processing fringepatterns images," Appl. Opt. 38, 788-794 (1999).

13. J. L. Marroquin, M. Servin, and R. Rodriguez-Vera, "Adaptive quadrature filters for the recovery of phase from fringe pattern images," J. Opt. Soc. Am. A 14, 1742-1753 (1997).

14. J. L. Marroquin and M. Rivera, "Quadratic regularization functionals for phase unwrapping," J. Opt. Soc. Am. A 12, 2393-2400 (1995).

15. J. L. Marroquin, M. Servin, and R. Rodriguez-Vera, "Adaptive quadrature filters for multiple phase-stepping images," Opt. Lett. 23, 238-240 (1998).

16. M. Servin, J. L. Marroquin, and F. J. Cuevas, "Demodulation of a single interferogram by use of a two-dimensional regularized phase-tracking technique," Appl. Opt. 36, 4540-4548 (1997).

17. B. Ströbel, "Processing of interferometric phase maps as complex-valued phasor images," Appl. Opt. 35, 2192-2198 (1996).

18. P. S. Theocaris and E. E. Gdoutos, Matrix Methods in Photoelasticity (Springer-Verlag, Berlin, 1979).

19. J. A. Quiroga and A. González-Cano, "Stress separation from photoelastic data by a multigrid method," Meas. Sci. Technol. 9, 1204-1210 (1998).

20. Matlab Optimization Toolbox, User's Guide, version 5 (MathWorks, New York, 1997). 